\title{
Pituitary-Adrenal Axis Responses to Routine Management Procedures in Sheep (Ovis aries)
}

\author{
Esterina Fazio, Pietro Medica, Cristina Cravana \& Adriana Ferlazzo
}

\begin{abstract}
Background: Shearing is considered as a stressful factor in sheep, capable of causing some behavioural changes including variations of body condition and hormonal and haematochemical parameters. On these basis, circulating ACTH and cortisol concentrations were determined in sheep before and after exposure to aversive stimuli in order to evaluate the potential use of these hormones to objectively measure physical and mental stress responses to isolation, restraint and shearing.

Materials, Methods \& Results: Forty Pinzirita sheep, used as experimental group, were evaluated in baseline conditions, 24 $\mathrm{h}$ before isolation, restraint and shearing (T0), after isolation (T1), after restraint and shearing (T2), by taking into account the influence of previous shearing experience (just shorn). A control group of forty sheep (intact) was evaluated in baseline condition (T0). According to previous experience the experimental subjects were divided in two groups, respectively group A represented by 20 sheep (10 males and 10 females), aged 1-2 years, without previous shearing experience (intact); and group B represented by 20 sheep (10 males and 10 females), aged older than 2 years, previously exposed to shearing (just shorn). A control group $\mathrm{C}$ of 40 sheep splitted into two sub-groups represented by 10 intact males and 10 intact females, aged 1-2 years, and 10 males and 10 females just shorn, aged older than 2 years, was evaluated in baseline conditions (T0). Blood samples were collected $24 \mathrm{~h}$ before experimental practices (T0: 08:00 h), after $1 \mathrm{~h}$ after of isolation (T1) and after restraint and shearing, around an average of $5 \mathrm{~min}$ (T2). All samples were taken in quiet conditions by the same operator. Serum ACTH concentrations were assayed by a commercial radioimmunoassay (RIA). Serum cortisol concentrations were assayed by amplified Enzyme Immunoassay (EIA). Two-way RM ANOVA showed a significant effect of husbandry procedures on ACTH changes, with higher ACTH and cortisol concentrations of just shorn than intact males $(\mathrm{F}=15.76$; $P<0.0001)$ and females $(\mathrm{F}=17.34 ; P<0.0005)$, and on cortisol changes, with higher cortisol concentrations of just shorn than intact males $(\mathrm{F}=6.54 ; P<0.01)$ and females $(\mathrm{F}=22.66 ; P<0.0001)$.

Discussion: The highest ACTH and cortisol values after isolation, with an appreciable increase especially after restraint and shearing in just shorn sheep, showed that the previous shearing exposure could not induce a habituation to the stimulus, probably promoting a larger release of ACTH and cortisol hormones, so inducing an early and marked coping response to stress in just shorn specimens. Thus, it would appear that different ACTH and cortisol responses of sheep with or without previous shearing experience over the study period resulted in some significant differences when exposed to isolation, restraint and shearing, probably due to changes in pituitary-adrenal axis reactivity. Nevertheless, it is possible to interpret the increase in $\mathrm{ACTH}$ and cortisol concentrations related to intrinsic endocrine control mechanisms as an increase due to a rise in the stressful stimulus or coping response. The magnitude of ACTH and cortisol changes following isolation, restraint and shearing suggests that measurements of both hormones are useful in assessing husbandry procedures stress in sheep, with marked elevations in just shorn sheep, indicating that experience can induce an early much larger coping response to stressful practice of shearing.
\end{abstract}

Keywords: sheep, restraint, shearing, ACTH, cortisol. 


\section{INTRODUCTION}

It is well established that animals respond to unpredictable aversive events by increasing output of hormones, such as adrenocorticotropin hormone (ACTH) and cortisol, into the bloodstream $[2,6,8,11,20]$. The pain, castration, weaning and isolation stressors markedly elevated plasma ACTH and cortisol concentrations in lambs [20] and sheep [15], and the amplitude of hormone response might correlate with the severity of the stimulus [24]. What is more, female sheep exhibited higher levels of plasma cortisol in response to isolation than males $[16,17,26]$, and during tests involving novelty or approaching avoiding a human [1]. There is evidence that the absence of visual and tactile contacts with conspecifics is the main factor influencing the stress response to social isolation [3], and lambs returned with a caregiver, after a period of social isolation, showed a more pronounced calming effect in individuals that were regularly handled [25].

Mears et al. [21] showed that the stress response to handling was equivalent to that of handling plus shearing, and that this response was much greater in ewes previously exposed to annual shearing, with the highest cortisol values. Moreover, both shearing and handling resulted in short-term elevated cortisol levels $[2,13,18]$. The goal of this study was to determine the mechanisms involved in pituitary-adrenal axis response to physical and mental stress of sheep exposed to isolation, restraint and shearing, by taking into account the influence of previous exposure to shearing.

\section{MATERIALS AND METHODS}

\section{Experimental design}

The study was carried out on eighty Pinzirita breed sheep belonging to a flock of about 200 animals. The farm was located in Sicily, at latitude: $37^{\circ} 56^{\prime} 1^{\prime \prime}$ $\mathrm{N}$, longitude: $13^{\circ} 39^{\prime} 58^{\prime \prime} \mathrm{E}, 550 \mathrm{~m}$ above sea level. Forty sheep ( 20 males and 20 females), used as experimental group, were evaluated in baseline conditions, $24 \mathrm{~h}$ before isolation, restraint and shearing (T0), after isolation (T1) and after restraint and shearing (T2), by taking into account the influence of the previous shearing experience. Shearing and relative associated husbandry procedures lasted around an average of 5 min per sheep. Experimental sheep were penned separately from the rest of the flock in the sheep barn, $24 \mathrm{~h}$ before to shearing, with visual, olfactory and acoustic contact with flock. Shearing was done on one day.

According to previous experience the experimental subjects were divided in two groups, respectively group A represented by 20 sheep (10 males and 10 females), aged 1-2 years, without previous shearing experience (intact); and group B represented by 20 sheep ( 10 males and 10 females), aged older than 2 years, previously exposed to shearing (just shorn). A control group $\mathrm{C}$ of 40 sheep splitted into two sub-groups represented by 10 intact males and 10 intact females, aged 1-2 years, and 10 males and 10 females just shorn, aged older than 2 years, was evaluated in baseline conditions (T0). During the day, the animals grazed on the natural pasture, and were penned in the night, when they received a commercial concentrated food-supplement of $200 \mathrm{~g}$ per head of crude protein (CP) $20.4 \%$ plus metabolizable energy MJ (ME) $12.5 / \mathrm{kg}$ dry matter. The sheep also had free access to hay (CP $11.1 \%$ and $7.2 \mathrm{MJ} \mathrm{ME} / \mathrm{kg} \mathrm{DM}$ ), water were continuously available. According to the traditional techniques of Sicilian sheep breeding, the animals are usually shorn once a year, in late spring at the end of June.

The environmental temperature and humidity were recorded with a Hygrothermograph ST-50 ${ }^{1}$. The weather was mild but wet; the average environmental temperature and mean relative humidity values on the experimental days were $28^{\circ} \mathrm{C}$ and $60 \%$, respectively.

\section{Blood sampling}

Sheep were accustomed to clinical routines and blood sampling; subjects were individually held in a corner of this pen, one at a time, and they were easily restrained by the same handler assistant who held their halter during collection of samples at T0 and T1. On the day of the experiment, a post-restraint and post-shearing (T2) blood samples were obtained with animals in lateral decubitus, with feet tied and lead rope.

All animals were sampled in the same order; thus, blood samples from control and experimental sheep were obtained at the same time of day for each group, via jugular venipuncture, to minimise the effect of circadian rhythms on ACTH and cortisol concentrations. Blood samples were collected $24 \mathrm{~h}$ before experimental practices (T0: 08:00 h), after $1 \mathrm{~h}$ after of isolation (T1) and after restraint and shearing, around 
an average of 5 min (T2). All samples were taken in quiet conditions by the same operator.

\section{Analysis of ACTH and cortisol}

Immediately after withdrawal, blood samples were refrigerated at $4{ }^{\circ} \mathrm{C}$ and were subsequently (within $1 \mathrm{~h}$ ) centrifuged for $15 \mathrm{~min}$ at $1,500 \times \mathrm{g}$. Serum was harvested and stored in polystyrene tubes at $-20^{\circ} \mathrm{C}$ and assayed for ACTH and cortisol analyses.

Serum ACTH concentrations were assayed by a commercial radioimmunoassay (RIA) following the instructions of the manufacturer kit (ELSA-ACTH) ${ }^{2}$. The limit of sensitivity of the ACTH assay was 0.44 pmol/L. The intra- and inter-assay CVs were 6 and $15 \%$, respectively.

Serum cortisol concentrations were assayed by amplified Enzyme Immunoassay (EIA) following the instructions of the manufacturer (SEAC/RADIM, Florence, Italy) ${ }^{3}$. The limit of sensitivity of the cortisol assay was $13.80 \mathrm{nmol} / \mathrm{L}$. The intra- and inter-assay CVs were 4.6 and $6.9 \%$, respectively.

\section{Statistical analyses}

A one-way repeated measures analysis of variance (RM ANOVA) was applied to determine whether isolation, restraint and shearing have any effect on hormonal variables. Results were analyzed by a repeated measures analysis of variance performed using a statistical analysis software programme (PRISM) ${ }^{4}$. Three sampling points (T0, T1, T2) were used to compare the treatments by the repeated measures analysis. A two-way repeated measures analysis of variance (2-way RM ANOVA) was applied to test the effect of isolation, restraint and shearing procedures and time on hormonal changes, as well as for the interaction between them, on hormonal concentrations. When the F statistic was significant, the differences between individual means over time were then assessed using a post hoc multiple comparison test (Bonferroni). Linear regression and correlation between ACTH and cortisol concentrations were also calculated. Statistical significance was set at $P<0.05$.

\section{RESULTS}

Results are expressed as means \pm standard error (se) for ACTH (Table 1) and cortisol (Table 2) concentrations.

Compared to control values, no significant differences between males and females, nor between just shorn and intact animals were obtained for $\mathrm{ACTH}$ values.

Compared to T0 values (baseline conditions), males showed higher ACTH values after isolation, at T1 (intact: $P<0.01$; just shorn: $P<0.001$ ), and after restraint and shearing, at T2 (intact: $P<0.01$; just shorn: $P<0.0001$ ); likewise, females showed higher ACTH values after isolation, at T1 (intact: $P<0.01$; just shorn: $P<0.0001$ ), and after restraint and shearing, independently of previous shearing experience, at T2 $(P<0.001)$.

Compared to $\mathrm{T} 1$ values (after isolation), males showed higher ACTH values after restraint and shearing, at T2 (intact: $P<0.001$; just shorn: $P<0.0001$ ); likewise, females showed higher ACTH values after restraint and shearing, at T2 (intact: $P<0.001$; just shorn: $P<0.0001)$.

One-way RM ANOVA showed a significant effect of husbandry procedures (isolation, restraint and shearing): on ACTH values of males (intact: $\mathrm{F}=$ 6.967; $P<0.005$; just shorn: $\mathrm{F}=26.67 ; P<0.0001$ ) and females (intact: $\mathrm{F}=14.87 ; P<0.0001$; just shorn: $\mathrm{F}=36.25 ; P<0.0001)$.

Two-way RM ANOVA showed a significant effect of husbandry procedures on ACTH changes, with higher ACTH concentrations of just shorn than intact males $(\mathrm{F}=15.76 ; \mathrm{P}<0.0001)$ and females $(\mathrm{F}=$ 17.34; $P<0.0005)$, of just shorn females than males $(\mathrm{F}=7.2 ; P<0.01)$ and of intact females than males $(\mathrm{F}=11.11 ; P<0.005)$.

Compared to control values, no significant differences between males and females, nor between just shorn and intact animals were obtained for cortisol values.

Compared to T0 values, males, independently of previous shearing experience, showed higher cortisol values after isolation, at T1 $(P<0.01)$ and after restraint and shearing, at T2 (intact: $P<$ 0.001; just shorn: $P<0.0001$ ); likewise, females showed higher cortisol values after isolation, at T1 $(P<0.001)$, and after restraint and shearing, at T2 $(P<0.0001)$.

Compared to $\mathrm{T} 1$ values, males showed higher cortisol values after restraint and shearing, at T2 (intact: $P<0.01$; just shorn: $P<0.001$ ); likewise, females, independently of previous shearing experience, showed higher cortisol values after restraint and shearing, at T2 $(P<0.001)$. 
One-way RM ANOVA showed a significant effect of husbandry procedures (isolation, restraint and shearing): on cortisol of males (intact: $\mathrm{F}=32.17$; $P<0.0001$; just shorn: $\mathrm{F}=30.92 ; P<0.0001)$ and females (intact: $\mathrm{F}=19.56 ; P<0.0001$; just shorn: $\mathrm{F}=$ 58.60; $P<0.0001)$.

Two-way RM ANOVA also showed a significant effect of husbandry procedures on cortisol changes, with higher cortisol concentrations of just shorn than intact males $(\mathrm{F}=6.54 ; P<0.01)$ and females (F $=22.66 ; P<0.0001)$, of just shorn females than males $(\mathrm{F}=93.95 ; P<0.0001)$ and of intact females than males $(\mathrm{F}=6.21 ; P<0.01)$.

No linear regression and correlation between ACTH and cortisol concentrations were observed.

Table 1. Circulating ACTH levels (mean \pm se) of sheep in control and in experimental groups before exposure to isolation, restraint and shearing (T0), after isolation (T1), after restraint and shearing (T2).

\begin{tabular}{|c|c|c|c|c|}
\hline \multicolumn{5}{|c|}{ ACTH (pmol/L) } \\
\hline & intact & just shorn & intact & just shorn \\
\hline & \multicolumn{2}{|c|}{ males } & \multicolumn{2}{|c|}{ females } \\
\hline control & $64.87 \pm 8.77$ & $62.27 \pm 8.52$ & $70.12 \pm 3.88$ & $69.78 \pm 8.04$ \\
\hline T0 & $61.15 \pm 6.03$ & $60.34 \pm 7.62$ & $69.50 \pm 8.34$ & $72.96 \pm 9.07$ \\
\hline $\mathrm{T} 1$ & $90.20 \pm 1.70^{\mathrm{a}}$ & $129.04 \pm 4.74^{\mathrm{b}}$ & $109.68 \pm 8.21^{\mathrm{a}}$ & $176.04 \pm 8.00^{c}$ \\
\hline $\mathrm{T} 2$ & $135.99 \pm 8.70^{\mathrm{aB}}$ & $263.61 \pm 8.28^{\mathrm{cC}}$ & $208.20 \pm 8.61^{\mathrm{cB}}$ & $318.30 \pm 9.70^{c C}$ \\
\hline
\end{tabular}

Different letter superscripts in columns show significant differences versus T0 values ( $\mathrm{a}=P<0.01 ; \mathrm{b}=P<0.001 ; \mathrm{c}=P<0.0001$ ), and versus T1 values $(\mathrm{A}=P<0.01 ; \mathrm{B}=P<0.001 ; \mathrm{C}=P<0.0001)$.

Table 2. Circulating cortisol levels (mean \pm se) of sheep in control and in experimental groups before exposure to isolation, restraint and shearing (T0), after isolation (T1), after restraint and shearing (T2).

\begin{tabular}{|c|c|c|c|c|}
\hline \multicolumn{5}{|c|}{ Cortisol (nmol/L) } \\
\hline & intact & just shorn & intact & just shorn \\
\hline & \multicolumn{2}{|c|}{ males } & \multicolumn{2}{|c|}{ females } \\
\hline control & $34.64 \pm 4.97$ & $51.83 \pm 8.40$ & $30.77 \pm 5.67$ & $68.69 \pm 8.54$ \\
\hline T0 & $32.99 \pm 6.51$ & $57.84 \pm 6.76$ & $32.05 \pm 7.51$ & $78.81 \pm 7.72$ \\
\hline $\mathrm{T} 1$ & $79.13 \pm 6.38^{\mathrm{a}}$ & $105.96 \pm 5.46^{\mathrm{a}}$ & $109.59 \pm 2.10^{\mathrm{b}}$ & $147.87 \pm 4.35^{\mathrm{b}}$ \\
\hline $\mathrm{T} 2$ & $118.17 \pm 2.18^{\mathrm{bA}}$ & $195.70 \pm 8.76^{\mathrm{cB}}$ & $173.17 \pm 4.75^{\mathrm{cB}}$ & $233.62 \pm 5.15^{\mathrm{cB}}$ \\
\hline
\end{tabular}

Different letter superscripts in columns show significant differences versus T0 values $(\mathrm{a}=P<0.01 ; \mathrm{b}=P<0.001 ; \mathrm{c}=P<0.0001)$, and versus $\mathrm{T} 1$ values $(\mathrm{A}=P<0.01 ; \mathrm{B}=P<0.001 ; \mathrm{C}=P<0.0001)$.

\section{DISCUSSION}

The adrenocortical response to stress was frequently measured in ruminants, with elevated values after either physical or psychological stress $[5,7,9,14]$. Hence, the comparison of our data, related to ACTH and cortisol concentrations of sheep, with published data for ovine species (Cortisol: 74 - $209 \mathrm{nmol} / \mathrm{L}$ [21]; ACTH: 8 - 12 pmol/L [23]; cortisol: 43 - $127 \mathrm{nmol} / \mathrm{L}$ [4]; ACTH: 27 - $36 \mathrm{pmol} / \mathrm{L}$; cortisol: 53 - $144 \mathrm{nmol} / \mathrm{L}$; [19]); revealed that ACTH and cortisol concentrations were in agreement with these physiological wide ranges. Nevertheless, slight variations might be ascribed to differences in methods and are the subject of speculation; in addition, some differences may also be explained by different experimental conditions, as the nature and severity of the procedure $[18,24]$.

Compared to control values, at $\mathrm{T} 0$ in baseline conditions ACTH and cortisol values did not show significant differences, irrespective of previous experience and sex. Likewise no significant differences were observed at $\mathrm{T} 0$ between just shorn and intact males, just shorn and intact females and between males and females, according to previous experience. These findings showed that baseline ACTH and cortisol concentrations were not affected by sex and previous experience, especially considering that both 
hormonal concentrations were similar in males and females. On the other hand, the observation that, in terms of circulating ACTH and cortisol responses, sheep previously exposed to annual shearing were stressed to a much greater extent than inexperienced sheep, confirms previous data reported by Mears et al. [21] in ewes. Likewise, these data confirm the cortisol response always observed in wethers that, when were exposed to the same stressors every 2 weeks, showed less adaptation resulting in much larger elevations in plasma cortisol concentrations [13] than those repeatedly stressed on a daily basis [22].

The marked increase of ACTH and cortisol responses in just shorn male and female specimens showed that sheep suffer shearing procedures, while the mechanism adopted to cope with this negative stress was more marked in just shorn and intact females than males, as showed by 2-way RM ANOVA. These results confirm the existence of sex differences in cortisol secretion after administration of an ACTH analogue in sheep (corresponding to stressful stimuli), with higher cortisol levels in ewes than rams [27]. Additionally, the concomitant ACTH and cortisol increase trends could be interpreted as a rise in perceived early isolation stimulus severity, with a marked increase after restraint plus shearing, probably due to permanent stress coping mechanisms. What is more, this was interpreted as an upward trend in perceived stimulus severity or no habituation to the stimulus, hence sheep, independently of previous experience and sex, were considered stressed.

It is well known that the effect of handling experience may depend on the age at which the handling occurs, and it may be possible to exploit the sensitivity of sheep to learning at certain ages; habituating sheep to handling routines, familiarizing them with yard configurations and training leader sheep at critical learning periods may improve later handling [12]. Nevertheless, in the present study the visual, chemical and acoustic communications with conspefics probably did not reduce physical and mental stress at certain levels also in just shorn animals, as well as in intact ones, partly because the animals were usually shorn once a year. These data suggest that ACTH and cortisol equally contribute to response to stressful husbandry procedures by increasing output of both hormones, as previously obtained in ewes after isolation stress test [19], and after isolation from the flock with or without lambs [15], although no significant correlation was obtained. Another point is that the stress response to isolation, restraint and shearing represented sufficient stimulus to affect pituitary-adrenal axis responsiveness. Stress is a multifactorial condition, and during routine livestock practices, many different stimuli can act as stressors. Hence, the combination of individual collative (previous experience, age) and management proceduresassociated (exposition to sounds, smells and sights of shearing operator) factors affects the pituitary-adrenal axis responses, which may influence the individual adaptation. Moreover, in this study, it was difficult to distinguish between physical and mental stress, and the lack of correlation between ACTH and cortisol changes in both intact and just shorn sheep was probably related to the different responses to physical and/ or mental stimuli, in agreement with previous study that suggests that novelty stimuli, during competition, increase ACTH and cortisol concentrations, without correlations between the two hormones [10].

Another point is that the magnitude of ACTH and cortisol changes following handling practices suggests that isolation, restraint and shearing represented progressive degree of stress in sheep, with the pituitary-adrenal axis response according to previous experience and sex. Moreover, the highest ACTH and cortisol values after isolation, with an appreciable increase especially after restraint and shearing in just shorn sheep, showed that the previous shearing exposure could not induce a habituation to the stimulus, probably promoting a larger release of ACTH and cortisol hormones, so inducing an early and marked coping response to stress in just shorn specimens.

\section{CONCLUSION}

Thus, it would appear that different ACTH and cortisol responses of sheep with or without previous shearing experience over the study period resulted in some significant differences when exposed to isolation, restraint and shearing, probably due to changes in pituitary-adrenal axis reactivity. Nevertheless, it is possible to interpret the increase in ACTH and cortisol concentrations related to intrinsic endocrine control mechanisms as an increase due to a rise in the stressful stimulus or coping response. 
MANUFACTURERS

${ }^{1}$ Sekonic Corporation. Tokyo, Japan.

${ }^{2}$ CIS BioInternational. Gif-sur-Yvette, France.

${ }^{3}$ SEAC/RADIM. Florence, Italy.

${ }^{4} \mathrm{GraphPad}$ Software Inc. San Diego, CA, USA.

Acknowledgements. Special thanks to veterinarians who participated in this study and to the farmers for taking care of the sheep.
Ethical approval. All procedures were formally approved by Ethical Committee for the care and use of animals of Department of Veterinary Sciences, and were performed in compliance with the guidelines of the Italian law on the care and use of animals (D.L. 4/3/2014 n. 26) and EU Directive (Directive 2010/63).

Declaration of interest. The authors declare that they have no conflict of interest that could be perceived as prejudicing the impartiality of the research reported.

\section{REFERENCES}

1 Boissy A., Bouix J., Orgeur P., Poindron P., Bibè B. \& Le Neindre P. 2005. Genetic analysis of emotional reactivity in sheep: effects of the genotypes of the lambs and of their dams. Genetics Seection Evolution. 37(4): 381-401.

2 Coulon M., Lévy F., Ravel C., Nowak R. \& Boissy A. 2014. Mild effects of gestational stress and social reactivity on the onset of mother-young interactions and bonding in sheep. Stress. 26(6): 1-40.

3 da Costa A.P., Leigh A.E., Man M.S. \& Kendrick K.M. 2004. Face pictures reduce behavioural, autonomic, endocrine and neural indices of stress and fear in sheep. Proceedings: Biological Sciences. 271(1552): 2077-2084.

4 Doyle R.E., Lee C., Deiss V., Fisher A.D., Hinch G.N. \& Boissy A. 2011. Measuring judgement bias and emotional reactivity in sheep following long-term exposure to unpredictable and aversive events. Physiology \& Behavior. 102(5): 503-510.

5 Fazio E., Medica P., Alberghina D., Cavaleri S. \& Ferlazzo A. 2005. Effect of long-distance road transport on thyroid and adrenal function and haematocrit values in Limousin cattle: Influence of body weight decrease. Veterinary Research Communications. 29(8): 713-719.

6 Fazio E., Medica P., Cravana C. \& Ferlazzo A. 2011. Adrenocortical response of jennies to milking stress. Livestock Science. 137(1-3): 278-281.

7 Fazio E., Medica P., Mignacca S., Cravana C. \& Ferlazzo A. 2011. Haematological and cortisol changes after 3 h road journey in sheep. Journal of Animal and Veterinary Advances. 10(19): 2487-2492.

8 Fazio E., Medica P., Cravana C., Cavaleri S. \& Ferlazzo A. 2012. Effect of temperament and prolonged transportation on endocrine and functional variables in young beef bulls. Veterinary Record. 171(25): 644.

9 Fazio E., Medica P., Cravana C. \& Ferlazzo A. 2014. Short- and long-term effects of weaning on adrenocortical and functional response of lambs. Acta Scientiae Veterinariae. 42: 1193.

10 Ferlazzo A., Medica P., Cravana C. \& Fazio E. 2012. Circulating $\beta$-endorphin, adrenocorticotropin, and cortisol concentrations of horses before and after competitive show jumping with different fence heights. Journal of Equine Veterinary Science. 32(11): 740-746.

11 Foreman H. \& Ferlazzo A. 1996. Physiological responses to stress in the horse. Pferdeheilkunde. 12(4): 401-404.

12 Hargreaves A.L. \& Hutson G.D. 1997. Handling systems for sheep. Livestock Production Science. 49(2): 121-138.

13 Hargreaves A.L. \& Huston G.D. 1990. Some effects of repeated handling on stress responses in sheep. Applied Animal Behaviour Science. 26(3): 253-265.

14 Hashizume T., Haglof S.A. \& Malven P.V. 1994. Intracerebral methionine-enkephalin, serum cortisol, and serum $\beta$-endorphin during acute exposure of sheep to physical or isolation stress. Journal of Animal Science. 72(4): 700-708.

15 Hasiec M., Tomaszewska-Zaremba D. \& Misztal T. 2014. Suckling and salsolinol attenuate responsiveness of the hypothalamic-pituitary-adrenal axis to stress - focus on catecholamines, $\mathrm{CRH}, \mathrm{ACTH}$, cortisol and prolactin secretion in lactating sheep. Journal of Neuroendocrinology. 26(12): 844-852.

16 Hernandez C.E., Matthews L.R., Oliver M.H., Bloomfield F.H. \& Harding J.E. 2009. Effects of sex, litter size and periconceptional ewe nutrition on the ewe-lamb bond. Applied Animal Behaviour Science. 120(1-2): 76-83.

17 Hernandez C.E., Matthews L.R., Oliver M.H., Bloomfield F.H. \& Harding J.E. 2010. Effects of sex, litter size and periconceptional ewe nutrition on offspring behavioural and physiological response to isolation. Physiology \& Behavior. 101(5): 588-594.

18 Jephcott E.H., McMillen I.C., Rushen J.P. \& Thorburn G.D. 1987. A comparison of the effects of electroimmobilisation and, or, shearing procedures on ovine plasma concentrations of beta-endorphin/beta-lipoprotein and cortisol. Research in Veterinary Science. 43(1): 97-100. 
19 Long N.M., Nathanielsz P.W. \& Ford S.P. 2012. The impact of maternal overnutrition and obesity on hypothalamicpituitary-adrenal axis response of offspring to stress. Domestic Animal Endocrinology. 42(4): 195-202.

20 Mears G.J. \& Brown F.A. 1997. Cortisol and $\beta$-endorphin responses to physical and psychological stressors in lambs. Canadian Journal of Animal Science. 77(4): 689-694.

21 Mears G.J., Brown F.A. \& Redmond L.R. 1999. Effects of handling, shearing and previous exposure to shearing on cortisol and beta-endorphin responses in ewes. Canadian Journal of Animal Science. 79(1): 35-38.

22 Minton J.E., Coppinger T.R., Reddy P.G., Davis W.C. \& Blecha F. 1992. Repeated restraint and isolation stress alters adrenal and lymphocyte functions and some leukocyte differentiation antigen in lambs. Journal of Animal Science. 70(4): 1126-1132.

23 Ramadoss J., Tress U., Chen W.J. \& Cudd T.A. 2008. Maternal adrenocorticotropin, cortisol, and thyroid hormone responses to all three-trimester equivalent repeated binge alcohol exposure: ovine model. Alcohol. 42(3): 199-205.

24 Smith R.F. \& Dobson H. 2002. Hormonal interactions within the hypothalamus and pituitary with respect to stress and reproduction in sheep. Domestic Animal Endocrinology. 23(1-2): 75-85.

25 Tallet C., Veissier I. \& Boivin X. 2009. How does the method used to feed lambs to modulate their affinity to their human caregiver? Applied Animal Behaviour Science. 119(1-2): 56-65.

26 Turner A.I., Canny B.J., Hobbs R.J., Bond J.D., Clarke I.J. \& Tilbrook A.J. 2002. Influence of sex and gonadal status of sheep on cortisol secretion in response to ACTH and on cortisol and LH secretion in response to stress: importance of different stressors. Journal of Endocrinology. 173(1): 113-22.

27 van Lier E., Pérez-Clariget R. \& Forsberg M. 2003. Sex differences in cortisol secretion after administration of an ACTH analogue in sheep during the breeding and non-breeding season. Animal Reproduction Science. 79(1-2): 81-92. 